\title{
Prevalence and associated factors of soil transmitted helminthes infestation among preschool children of Vadamaradchi Educational Zone
}

\author{
R Karunaithas ${ }^{1}$, A Murugananthan ${ }^{2}$ and S Kannathasan ${ }^{2, \#}$ \\ ${ }^{1}$ Medical Laboratory Sciences, Allied health Sciences, Faculty of Medicine, University of Jaffna \\ ${ }^{2}$ Division of Parasitology, Department of Pathology, Faculty of Medicine, University of Jaffna
}

Received: 6 October 2011

In Final Format: 3 February 2012

This cross sectional study was designed to find out the prevalence of soil transmitted helminth infestation and associated factors among preschool children of Vadamaraadchi Educational Zone in Jaffna district. One preschool was randomly selected from each division (3) of the educational zone, and all the children $(n=103)$ of selected schools were included in the study. Faecal samples were collected and examined by direct wet smear, salt flotation and formal ether concentration techniques. Demographic, socio-economic and health related behavior data were obtained from the parents using interviewer administered questionnaire. Prevalence of soil transmitted helminthes was zero in this study. Majority of the fathers were fishermen (55\%), whereas most of the mothers were housewives (86\%). Sixty five percentage (65\%) of fathers and $68 \%$ of mothers studied up to G.C.E O/L. Eighty nine (86\%) parents were aware of worm infestation. Ninety nine (96\%) stated that they cleaned their children after defecation. Among them, the majority always (88\%) and a few (8\%) sometimes washed their hands with soap and water after cleaning. Almost an equal proportion of children used water sealed toilets (48\%) and open ground (47\%) for defecation. Fifty two (50\%) children always and $43(42 \%)$ children sometimes used foot ware when they went out. Most of the children (96\%) received antihelminthic drugs. Seventy two children (70\%) received the drug within the last 3 months. The study indicates, the reduction in the prevalence of soil transmitted helminthes could be mainly due to anti-helminthic prophylaxis and practice of hand washing by the parents. A case control study has to be carried out to confirm the above conclusion. The majority (70\%) received more frequent anti helminthic prophylaxis. As there are reports of anti helminthic resistance, further study is needed to find out the appropriate intervals at which anti-helminthic prophylaxis to be given.

Key words- Soil Transmitted Helminthes, preschool children, antihelminthic prophylaxis

"Division of Parasitology, Department of Pathology, Faculty of Medicine, University of Jaffna Email-selvamkannathasan@gmail.com.T.P No-0212222073 
Introduction

Soil transmitted helminthiasis (STH) is one of the major causes of public health problems around the world, especially in developing countries, particularly in tropical regions (Ramsay et al., 1991). World Health Organization has estimated that Ascaris lumbricoides, Necator americanus / Ancylostoma duodenale and Trichuris trichiura infect 2.5 billion, 1.5 billion and 1.0 billion people worldwide, respectively (WHO, 1997).

Low socio-economic state and poor sanitation coupled with low educational rates of parents, particularly the mothers, are the main causes influencing the transmission and distribution of the infestation (Okyay et al., 2004). In addition, environmental, demographic and healthrelated behaviors also influence the infestation (Norhayati et al., 2003).

The morbidity of STH infestations is greatest among children of school age and may have an adverse effect on growth (Nematian et al., 2008). Preschool children, defined as aged less than five years, make up between $10 \%-20 \%$ of the two billion people worldwide. Among them, 21 million

\section{Materials and Methods}

\section{Study area:}

This study was carried out during June 2011 in Vadamarachy Educational zone, which is further divided into 3 smaller administrative divisions. One representative preschool was randomly selected from each division. All the students of those selected preschools were included in this study. were infested with Hookworm, 122 million were infested with $A$. lumbricoides and 86 million were infested with $T$. trichiura (De Silva et al., 2003).

STH are a common problem in Sri Lanka, a due to poor socio-economic conditions, unhygienic environment and poor knowledge regarding health (Pawlowski, 1985). High prevalence of intestinal parasitic infestation was observed in the slum area in Colombo (De silva and Jayatileka, 1981). General infestation rate was $73.0 \%$ for urban and $88.3 \%$ for rural areas of Sri Lanka (Jayewardene, 1957).

Intestinal parasitic infestation in children living in the under privileged sector of the Jaffna Municipality was 50\%, where whipworm infestation was found to be the highest (27\%) (Nageswaran and Sivarajah, 1986). After treatment with antihelminthics hook worm and whipworm infestations reappeared sooner than roundworm in a periurban population in Jaffna (Nageswaran and Sivarajah, 1989).

There are no recent studies in Jaffna district to assess the current prevalence of intestinal helminthes and its associated factors among preschool children.

\section{Ethical consideration:}

Ethical clearance for the study was obtained from the Ethical Review Committee of the Faculty of Medicine, University of Jaffna. Permission to carry out the study was also obtained from the Director of Education, Vadamarachi Zone and the respective teachers of the preschools.

Ving. Journal of Science, Vol. 10, No. 12011 
Data collection:

After obtaining written consent from the parent or guardian of the child, a pretested interviewer-administered structured questionnaire was used to collect data. The questions were translated into native language (Tamil). The questionnaire was composed of the following components a) demographic and socio ecconomic data b) health practices towards prevention of intestinal nematode infestation d) deworming history of the child.

\section{Collection of stool sample:}

Stool sample was collected using labeled, clean, dry, wide mouthed containers which were given to parents on the day previous to the day of the study, after thoroughly explaining the way of collection. Collected stool samples were transported to the laboratory of the division of Parasitology, Faculty of Medicine, University of Jaffna as soon as possible.

\section{Identification of ova:}

One drop of normal saline was placed on a clean slide and a small amount of stool sample ( $2 \mathrm{mg}$ ) was thoroughly mixed. A cover slip was placed on top of the sample. Then, it was observed under the microscope using low power $(\times 10)$ objective lens.
One drop of iodine solution was placed on the clean slide and small amount of stool sample ( $2 \mathrm{mg}$ ) was fully mixed. A cover slip was placed on top of the sample. Then, it was observed under the low power $(\times 10)$ objective of light microscope. One gram of stool was emulsified in the saturated salt $(\mathrm{NaCl})$ solution and the larger debris was removed. Then, the test tube was filled to the top with saturated salt solution until convex meniscus forms and a cover glass was laid over the top and covered with slide which was observed under low power objective $(\times 10)$ lens (WHO, 2003). One gram of stool sample was fixed in formalin and strained through gauze. Then, $0.85 \% \mathrm{NaCl}$ was added and centrifuged (500 X g for 2 mins). The supernatant was decanted the test tube was filled with formalin and centrifuged. Then, the sediment was mixed with 4-5ml Ethyl Acetate and formalin and mixed well and centrifuged. The supernatant was decanted and sediment was mixed and placed on the slide and covered with a cover slip and observed under low power objective $(\times 10)$ lens (WHO, 2003). Twenty five samples were cross checked by the technical officer of the division of Parasitology, Faculty of Medicine, University of Jaffna.

\section{Data analysis:}

Data were analyzed using SPSS statistical software version 17. 


\section{Results}

Prevalence of intestinal helminthes

None of the 103 stool samples containedova of soil transmitted helminths, using normal saline and iodine wet smear. This was further was confirmed by two concentration techniques, i.e., salt flotation and formal-ether concentration technique.
Demographic data of the parents

More than half of the fathers (55\%) of the children were fishermen and most of the mothers (86\%) were house wives. Majority of the fathers (65\%) and most of the mothers (68\%) studied up to G.C.E O/L (Table 1).

\begin{tabular}{llll}
\hline Variable & Category & \multicolumn{2}{c}{ Response (N=103) } \\
& & Fother \\
& & & \\
\hline Educational & No education & $01 \%$ & $01 \%$ \\
Level & Up to grade 5 & $22 \%$ & $07 \%$ \\
& From grade 6 to Grade 9 & $03 \%$ & $\%$ \\
& Studied up to G.C.E O/L & $65 \%$ & $68 \%$ \\
& G.C.E.A/L or above & $05 \%$ & $09 \%$ \\
& Graduate & $04 \%$ & $01 \%$ \\
\hline Occupation & Laborer & $12 \%$ & $08 \%$ \\
& Farmer & $00 \%$ & $00 \%$ \\
& Fisherman & $55 \%$ & $00 \%$ \\
& Unemployed / Housewife & $10 \%$ & $86 \%$ \\
& Private shop & $04 \%$ & $00 \%$ \\
& Teacher/Clerk & $02 \%$ & $06 \%$ \\
& Professional/Executive & $01 \%$ & $00 \%$ \\
& Others & $16 \%$ & $00 \%$ \\
\hline
\end{tabular}

Table 1: Educational and occupational status of parents of the preschool children

Health practices towards preventing soil transmitted helminthes

Eighty nine (86\%) parents stated that they were aware of worm infestation. Ninety nine parents (96\%) stated that they used to clean their children after defecation. Among them, majority (88\%) always and few (8\%) sometimes washed their hands with soap and water after cleaning. An almost equal proportion of children used water sealed toilet $(48 \%)$ and open air (47\%) for defecation. Fifty two (50\%) children always and 43 (42\%) children sometimes used foot ware when they go out (Table 2). 


\begin{tabular}{lll}
\hline Variable & category & $\begin{array}{l}\text { Response } \% \\
(\mathrm{~N}=103)\end{array}$ \\
\hline \multirow{2}{*}{$\begin{array}{ll}\text { Washing hands with soap and water } \\
\text { after cleaning the defecated child }\end{array}$} & Always & $90 \%$ \\
\hline \multirow{3}{*}{ Toilet facility } & Never & $08 \%$ \\
& Water sealed toilet & $48 \%$ \\
\hline \multirow{3}{*}{ Wearing slippers while going out } & Open air defecation & $47 \%$ \\
& other & $05 \%$ \\
\hline & Always & $50 \%$ \\
& Some times & $42 \%$ \\
& Never & $08 \%$ \\
\hline
\end{tabular}

Table 2: Hygienic habits useful in preventing STH.

Source of antihelminthic drugs and period of deworming

Ninety eight (96\%) children have been given antihelminthic therapy. These drugs were obtained from Public Health
Inspectors (40\%), Government hospitals (28\%) or pharmacies $(27 \%)$. Seventy two children (70\%) received the drug within the last 3 months (Table 3).

\begin{tabular}{lll}
\hline Variable & Category & $\begin{array}{l}\text { Response } \% \\
(\mathrm{~N}=103)\end{array}$ \\
\hline \multirow{3}{*}{ Antihelminthic drugs given } & Yes & $96 \%$ \\
& No & $04 \%$ \\
\hline \multirow{3}{*}{ Period of deworming } & Within 3 months & $70 \%$ \\
& Within 3- 6 month & $25 \%$ \\
& Never given & $05 \%$ \\
\hline \multirow{3}{*}{ Source of deworming drug } & Public health & $40 \%$ \\
& Inspectors/Midwives & $28 \%$ \\
& Govt. Hospitals & $27 \%$ \\
\hline
\end{tabular}

Table 3: Anti helminthic prophylaxis practiced by the preschool children. 


\section{Discussion}

Intestinal parasitic infestations are endemic worldwide and a major public health problem in developing countries (Shakya et al., 2009). Many studies have been carried out in Pakistan (Ahmad Khan et al., 2004), Bangladesh (Farhana et al., 2007), India (Paul et al., 1999) and elsewhere in the world regarding intestinal helminthiasis.

In and around Colombo and the greater Colombo area of Sri Lanka the prevalence of intestinal parasitic infestation was reported as 90.8\% (Sivalingam, 1961). Another Sri Lankan study carried out in the Hindagala Community Health area (Malcolm et al., 1976) indicated a high prevalence $(83.5 \%)$ of infestations. Further, in Jaffna district, $50 \%$ infestation was observed with the high rate of roundworm infestation (22.5\%) in Jaffna Municipality area (Nageswaran and Sivarajah, 1986). Another study carried out in the pediatric ward of the General Hospital (Teaching) Jaffna also showed a high prevalence rate (67\%) of intestinal helminth infestation (Ramadas and Ramadas, 1989). A recent study carried out in 2010, among 145 subjects in a psychiatric institution in Sri Lanka, showed that 35\% were infested with at least one intestinal parasite (Chandrasena et al., 2010).

But, according to our study carried out in 2011, a zero prevalence of STH was observed in Vadamaradchchi Educational Zone, in Jaffna district. Due to the logistic reasons this study was carried out in a dry period. Further study should be carried out continuously for one year to rule out the effect of climate on antihelmithic transmission. Regarding the educational level, majority of the parents studied up to G.C.E. O/L (fathers $-65 \%$ and mothers 68\%). This reasonable intermediate educational level could have lead to a good awareness level (85 \%) of worm infestations. A similar finding was observed in a study carried out in Saudi Arabia (Nagwa and Magda, 2010). Another study carried out in Turkey, indicated that there was a strong association between mother's educational level and awareness of parasitic infestations (Okyay et al., 2004). On the contrary in Nepal, no significant differences were observed in parasite positivity rates in spite of relatively low literacy rates (Batu et al., 2004).

Studies carried out in Nepal (Gyawali et al., 2009) and in Pakistan (Ahmad Khan et al., 2004) have proved that the prevalence of intestinal helminthiasis was high among people going to the open fields for defecation. In this study, only $48 \%$ of respondents used water sealed toilet and $47 \%$ used open places for the defecation. Though almost equal amount of children used water sealed toilet and open place defecation, there were no difference in the prevalence was observed in this study. However, the usage of toilets and foot ware may be nullified by the high usage of antihelmithic prophylaxis.

This study shows the efficacy of frequent antihelmithic prophylaxis in reducing prevalence of intestinal nematodes, where other factors are masked higher prevalence rate of intestinal helminth infestation was observed in the group with inconsistent hand washing 
practices after defecation (Gyawali et al., 2009). In our study, $96 \%$ of the parents stated that they cleaned their children after defecation. Among them, the majority always (88\%) and a few (8\%) sometimes washed their hands with soap and water after cleaning. This may be one of the factors which could attributed towards reducing the prevalence of intestinal helminthiasis, in addition to the main factor i.e., frequent antihelmithic prophylaxis.

As mentioned in the previous study carried out in Nigeria (Houmsou et al., 2010), there was an association between wearing footwear and reductions of intestinal parasitic infestation. In this study, 52 (50\%) preschool children always and 43 $(42 \%)$ children sometimes wore footwear when they went out. This foot wearing habit also has little impact on the prevalence of the intestinal nematodes. But, the impact of wearing foot ware could have been masked by the frequent antihelmithic prophylaxis.

The most important precautionary measure observed in our study was antihelminthic prophylaxis. The regular intake of deworming drugs (96\%), particularly $70 \%$ having been administered antihelminthics within 1-3months, could have reduced the worm infestations in the present study. The deworming tablets were obtained from $\mathrm{PHI}$ (40\%), hospital (28\%) and pharmacy (27\%).

\section{Acknowledgments}

Authors acknowledge Dr. A. Ketheewaran, Regional Director of Health Service, Northern Province, Jaffna, and Mr. S. Thillainathan, Assistant Director of
Other studies in Vietnam (Phuong et al., 2006) and Sri Lanka (Fernando et al., 2001, de Silva, 2003, De silva et al., 2003) have showed the efficacy of frequent administration of anthelminthics in reducing transmission. These results indicate the positive impact of deworming on the reduction of STH. Parents obtain anthelminthic therapy from private pharmacies in addition to the drugs administered by the government health sector. This reveals a high awareness among parents about the efficacy of antihelminthic therapy in reducing worm infestation. In conclusion, the zero prevalence of STH among preschool children in Vadamaarachy Educational Zone was most likely to be due to frequent antihelminthic prophylaxis and practice of hand washing by the parents.

De Silva et al reported that in areas of very high prevalence, thrice-yearly mass chemotherapy probably improves health better than twice-yearly treatment (De Silva et al, 2003). At the same time, there are reports for anti helminthic resistance in Belgium (Geerts and Gryseels, 2001) and in Mali (De Clercq et al., 1997). Hence, the question arises, "is the anti helminthic prophylaxis used in a rational manner?" Therefore, further study should be carried out to find out the interval in which the anti-helminthic prophylaxis to be given.

Education (ECD), Vadamaraadchi Educational Zone for permission to conduct this research. We also acknowledge Mr. Y. Sivagnanachelvan, Division of Parasitology, Department of Pathology, Faculty of Medicine, University of Jaffna, for 
laboratoryassistance. PHIs and Midwives of the Point Pedro and Karavetti $\mathrm{MOH}$ office for their assistance. Teachers and parents for their permission and cooperation in collecting the stool sample and completing the questionnaires.

\section{References}

1. Ahmad Khan, Abida Sultana, Abdul Majid Khan Dar, Haroon Rashid and Syed Abdul Ahad Najmi. (2004). A study of prevalence, distribution and risk factors of intestinal helmithic infestation in district Bagh (Azad Kashmir). Pakistan Armed Force Med J 8(1): 14-17

2. Batu Krishna Sharma, Shiba Kumar Rai, Diyo Ram Rai and Deepali Roy Choudhury. (2004).Prevalence of Intestinal Parasitic infection in school children in the Northeastern part of Kathmandu Valley, Nepal. Southeast Asian J Tropl Med Public Health 35(3): 501 -505.

3. Chandrasena T.G., Balasooriya B.A., Imbulpitiya I.V. and de Silva N.R. (2010). A survey for intestinal parasites in a psychiatric institution in Sri Lanka. Ann Trop Med Parasitol. 104(7): 605-608.

4. De Clercq D., Sacko M., Behnke J., Gilbert F., Dorny P. and Vercruysse J. (1997). Failure of mebendazole in treatment of human hookworm infections in the southern region of Mali. Am J Trop Med Hyg. 57(1): 25-30.

4. De Silva N.R. (2003). Impact of mass chemotherapy on the morbidity due to soiltransmitted nematodes. Acta Tropica. 86(23): 197-214.
5. De Silva N.R., Brooker S., Hotez P.J., Montresor A., Engels D. and Savioli L. (2003). Soil-transmitted helminth infections : updating the global picture. Trends in Parasitol 19(12): 547-551.

6. De silva D.G.H. and Jayatileka. (1981). Prevalence and severity of soil transmitted helminthes in an urban slum community in Colombo. Cey Med J 26: 160-164.

7. Muznebin F., Khanum $\mathrm{H}$ and Hossain A. (2007). Incidence of nematodes infections among the children brough to ICDDR, B Hospital, Dhaka, Bangladesh. J Bio Sci. 5: 159-164.

8. Fernando S.D., Goonethilleke H., Weerasena K.H., Kuruppuarachchi N.D., Tilakaratne D., De Silva D. and Wickremasinghe A.R. (2001). Geohelminthes infections in a rural area of Sri Lanka. Southeast Asian J Trop Med Public Health. 32(1): 23-26.

9. Gyawali N., Amatya R., Nepal HP. (2009). Intestinal parasitosis in school going children of Dharan municipality, Nepal. Trop Gastroenterol. 30(3):145-147.

10. Geerts S. and Gryseels B. (2001). Antihelmintic resistance in human helminthes: a review. Trop Med Inter Health 6(11): 915-921.

11. Houmsou, R.S., Amuta E.U. and Olusi T.A. (2010). Prevalence of intestinal parasites among primary school children in Makurdi, Benue State- Nigeria. The Internet Journal of Infectious Diseases. 8(1): 80-86. 
12. Jayewardena L.G. (1957). A study of parasitic infection in school children. Cey Med J. 4: 99.

13. Malcolm A., Fernando and Balasooriya S. (1976). Prevalence of soil transmitted helminthes in the Hindagala Community Health project area. Cey Med J. 22: 177183.

14. Nageswaran C. and Sivarajah N. (1986). Intestinal parasitic infestation in Children living in the under privileged sector of Jaffna municipality. Jaffna Med J. 21: 23-28. 15. Nageswaran C. and Sivarajah N. (1989). Re-infestation of intestinal nematodes in a peri- urban population, Jaffna. Jaffna Med J. 24: 81-84.

16. Nagwa S.M. Aly and Magda M.M. Mostafa. (2010). Intestinal parasitic infection among children in the kingdom of Saudi Arabia. Australian J Basic and Appl Sci. 4(9): 4200-4204.

17. Nematian J., Gholamrezanezhad A. and Nematian E. (2008). Giardiasis and other intestinal parasitic infections in relation to anthropometric indicators of malnutrition : a large,population-based survey of schoolchildren in Tehran. Ann Trop Med Parasitol. 102(3): 209-214.

18. Norhayati M., Fatmah M.S., Yusof S. and Edariah A.B. (2003). Intestinal parasitic infections in man. A review Med. J. Malaysia. 58(2): 296-305.

19. Okyay P., Ertug S., Gultekin B., Onen O. and Beser E. (2004). Intestinal parasites prevalence and related factors in school children, a western city sample-Turkey. BMC Public Health. 4:64.

20. Paul I., Gnanamani G. and NaUam N. (1999). Intestinal Helminth Infections Among School Children in Visakhapatnam. Indian J Pediatr. 66(5): 669-673.

21. Pawlowski Z. S. (1985). Ascriasis control. World Health Forum 6: 254-256.

22. Phuong H., Nguyen, Khan C Nguyen, Toan D Nguyen, Mai B Le, Caryn Bern Rafael Floresand Reynaldo Martorell. (2006). Intestinal helminth infestation among reproductive age women in Vietnam: Prevalence, co-infection and risk factors. Southeast Asian J Trop Med Public Health. 37(5): 1-10.

23. Ramadas G. and Ramadas D. (1989). Pattern of Parasites in a Pediatric ward in the General Hospital (Teaching), Jaffna. Cey J Child Health. 18: 29-33.

24. Ramsay A., Gillespic S.H., MnZava J.D.E., Ngo Wi F.I. and Fox R.A. (1991). Field evaluation of the formal detergent method for concentrating fecal parasites. J. Trop Med Hyg. 94: 211 -213.

25. Shakya B., Bhargava D., Shrestha S. and Rijal B.P. (2009). Intestinal parasitosis. J Institute of Medicine. 31(3): 13-16.

26. Sivalingam.V. (1961). An intestinal parasitic survey of an apparently healthy population in Ceylon. J Cey Public Health Assoc. 2: 23. 
27. WHO. (1997). Conquers suffering enriching humanity. The World Health Report. Geneva pp 15-19.
28. WHO. (2003). Parasitology. In: Manual of Basic Techniques for a health laboratory. $2^{\text {nd }}$ Edition. Geneva. pp 105-156. 